\title{
Tracheal stenosis, pulmonary agenesis, and patent ductus arteriosus
}

\author{
C. S. NELSON, I. K. R. MCMILLAN, AND \\ P. K. B H A R U C H A \\ From the Wessex Cardiac and Thoracic Centre, Southampton
}

Developmental anomalies of the major pulmonary tree are rare, but are now recognized more frequently with improved methods of investigation. They are usually associated with other congenital defects affecting principally the cardiovascular, skeletal, and urogenital systems, but as an isolated lesion they are very rare. Defects of the cardiovascular system occur more commonly with pulmonary malformations, and these include patent ductus arteriosus, septal defects, cor biloculare, and maldevelopments of the aortic arch and its great vessels.

A case of congenital tracheal stenosis, unilateral agenesis of the lung, and an associated patent ductus arteriosus is described together with the management.

\section{CASE REPORT}

Andrew H. was admitted on 22 November 1963 to the Southampton Children's Hospital three weeks after a full-term normal delivery. He had deep neonatal jaundice, which subsided after two weeks.

$\mathrm{He}$ developed a persistent cough, stridor, and laboured breathing the day before his admission. This was followed by cyanosis and bronchopneumonia, which responded to tetracycline. His admission radiograph is shown (Fig. 1).

About a month afterwards his respiratory symptoms again recurred and he was transferred to this unit on 12 March 1964. The radiograph showed no change.

On the same day a diagnostic bronchoscopy was performed under general anaesthesia to investigate the congenital stridor and the persistent abnormal chest radiographs, and this revealed two congenital malformations. The first was a localized tracheal stenosis, $2 \mathrm{~mm}$. in diameter, just below the level of the suprasternal notch; this was dilated to a size 14 French bougie. Retained secretions could then be aspirated, but the laboured breathing and stridor were not relieved. After about 45 minutes it became obvious that dilatation had not produced adequate relief. A tracheostomy at the level of the second and third tracheal cartilages was therefore performed.
Through this the child was again bronchoscoped with some difficulty. The second anomaly was then discovered and consisted of an absent left bronchus; the trachea at the expected site of the carina was deviated to the left, and at this site the curvature was greatest. A unilateral agenesis of the lung was then thought most likely, but gross stenosis of the left main bronchus could not be excluded. Further dilatation of the stenotic trachea and insertion of a silver tracheostomy tube relieved the difficult respiration and stridor when the tube had been passed just beyond the stenosis.

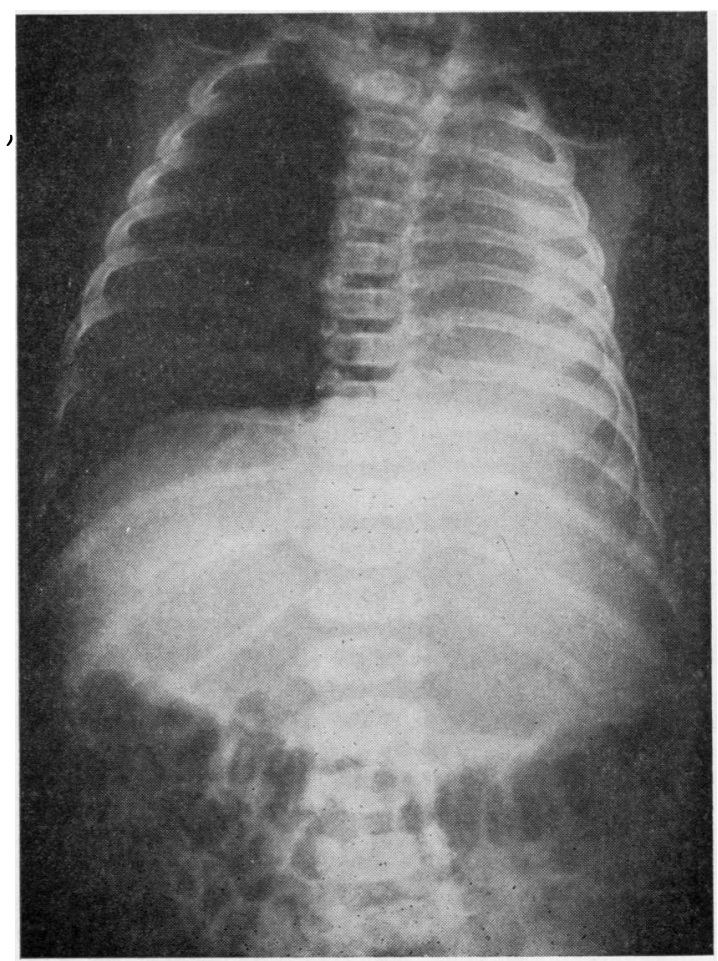

FIG. 1. Admission radiograph. 
As long as the tube remained in position the child breathed quietly, but if this was removed immediate respiratory difficulty occurred, which would have been fatal if not treated.

BRONCHOGRAPHY On 17 April 1964 a bronchogram (Fig. 2) was performed. It was done under general anaesthesia and the infant withstood it without any untoward effects. This showed certain interesting features: There was a post-stenotic dilatation of the trachea at the level of the carina. This dilatation also involved the right main bronchus. The right upper lobe

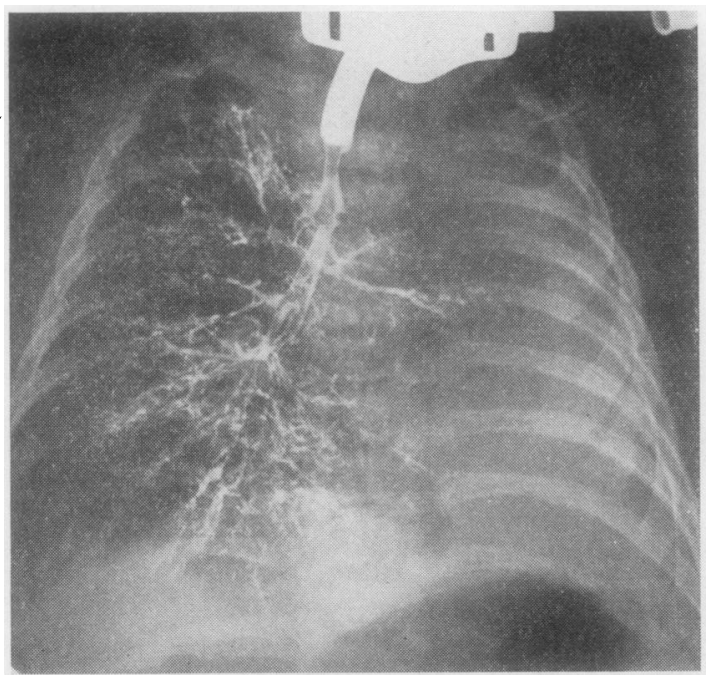

FIG. 2. P.A. bronchogram.

bronchus originated above or at the same level as the carina. A blind pouch, $0.5 \mathrm{~cm}$. in length, representing the left main bronchus was outlined. This bronchogram depicted herniation of the existing functioning lung across to the contralateral side. The herniation was formed chiefly by the posterior segment of the right upper lobe and the apical segment of the right lower lobe. The trachea was deviated to the left, as expected.

PATENT DUCTUS ARTERIOSUS This third developmental abnormality was discovered only when the child had lost his stridor; it was then possible to hear the typical murmur of patent ductus arteriosus.

The patent ductus arteriosus was divided at a left lateral thoracotomy on 20 March 1964. It had to be divided urgently because its presence had considerably increased the risks to which this infant was exposed. The child was exposed to frequent episodes of hypoxia for a varying length of time. The hypoxia caused an elevated pulmonary hypertension, and this caused the patent ductus arteriosus shunt to reverse completely or become bidirectional. It was therefore considered that the child could die during this period of desaturation caused by the reversed shunting.
There was complete absence of the left pleural cavity, the left lung, and the left pulmonary artery. The patent ductus arteriosus was $1.25 \mathrm{~cm}$. long and about $0.5 \mathrm{~cm}$. in diameter. This was divided and its ends were sutured. The single pulmonary artery was larger than expected for the infant's age. A search was made for vascular rings and other obliterated vascular structures. Another post-operative bronchoscopy was performed, and at the end of these surgical procedures the infant's condition was satisfactory.

MANAGEMENT OF THE TRACHEAL STENOSIS The child maintained improvement after division of the patent ductus arteriosus, and several attempts were made to wean him off his tracheostomy, but his respiratory symptoms returned on each occasion.

The management of the child with the tracheostomy tube in place was a complicated nursing problem. There was constant risk of the tube getting displaced, leading to respiratory obstruction and death (Fig. 3).

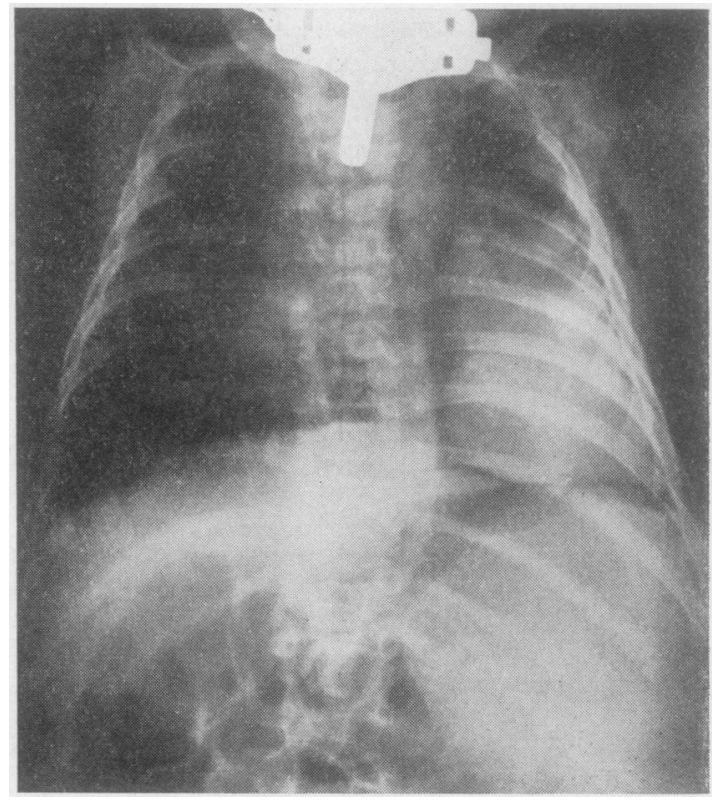

FIG. 3. Chest film after division of patent ductus arteriosus.

As this situation could not be maintained indefinitely it was decided to repair the tracheal stenosis.

On 8 April 1964 (six weeks after the division of the patent ductus arteriosus), through a right thoracotomy, under general anaesthesia, the tracheal stenosis was corrected by insertion of a dermal graft. Moderate hypothermia was induced by surface cooling, so that respiratory arrest could be maintained for an adequate period. The trachea was mobilized and opened longi- 
tudinally through the stenosed area. The dermal graft, $10 \mathrm{~mm}$. long by $4 \mathrm{~mm}$. wide and reinforced by a continuous ' $W$ ' stainless wire, was used to fill the tracheal gap. After completion of this repair an armoured latex tube was extended down through the tracheostomy to just above the orifice of the upper lobe bronchus.

The infant had a stormy passage during the first week after repair of the stenotic trachea. On one occasion he had a cardiac arrest preceded by hypercapnia and respiratory acidosis, but he recovered with treatment. The child subsequently made an excellent recovery but was kept in hospital for observation. One month after the insertion of the dermal graft he was found dead without any warning whatsoever. No satisfactory cause could be found for his death. Tracheostomy was maintained until death.

NECROPSY FINDINGS Necropsy showed no residual stenosis and no leak, but the graft was covered by a little mucopus. There was slight collapse of the right lung in the region where it was adherent to the thoracotomy incision. A small empyema cavity was present also in this area. No other congenital abnormality was seen.

\section{AETIOLOGY}

The factors responsible for this malformation are not understood. Apart from the one case of a father and daughter with identical right pulmonary hypoplasia, a collateral systemic arterial supply, and venous drainage (Neill, Ferencz, Sabiston, and Sheldon, 1960), no other case with a possible genetic cause has been reported. During a discussion on congenital abnormalities of the pulmonary vessels and malformations of the lung Ferencz (1961) noted that a few authors had drawn attention to the occurrence of lung hypoplasia and agenesis in twins (Sandoz, 1927; Finkelstein, 1912). It is possible that a genetic factor can be discounted even in twins with similar or dissimilar hypoplastic or agenetic lungs. Other factors are more likely to have affected the twins at the identical stage of their embryonic development. The association of trauma, miscarriage, and infections during the first three months of pregnancy and malformation of the major tracheo-bronchial tree has been stressed by Field (1946).

Lung agenesis has been produced in rats by placing the pregnant rats on a diet deficient in vitamin A (Wilson and Warkany, 1949). Viral agents may produce congenital abnormalities of the lung, as in congenital heart disease following rubella in pregnancy, but no firm evidence is available (Campbell, 1961; Butler, Dudgeon,
Hayes, Peckham, and Wybar, 1965 ; Forrester, Lees, and Watson, 1966).

\section{INCIDENCE}

Though pulmonary agenesis is rare compared with cardiovascular malformations, the true incidence may be higher than was originally thought. Wexels (1951) found 85 authentic cases in the world literature. Two of these were first reported by him. This subject was reviewed by JimenezMartinez, Perez-Alvarez, Perez-Trevino, RubioAlvarez, and de Rubens, in 1965, and an additional 33 cases were collected from various parts of the world. These authors credited Morgagni in 1762 with the first description of pulmonary agenesis. Both sexes seem to be equally affected. The majority of these cases were discovered at necropsy. More and more lung malformations are seen during life owing to improved diagnostic facilities and improved paediatric care. Only 18 of the 87 agenetic cases tabulated by Wexels (1951) were diagnosed during life.

\section{CLASSIFICATION AND PATHO-EMBRYOLOGY}

The universally accepted classification was first proposed by Schneider and Schwalbe in 1913, and this is subdivided into three main categories: bilateral complete agenesis; unilateral agenesis; and lobar agenesis and other lesser bronchial abnormalities. Bilateral complete agenesis is incompatible with life. It can be inferred that the factor or factors responsible for pulmonary agenesis act at an early stage on the foetus. The primitive respiratory apparatus first appears as a groove in the floor of the foregut at the $3 \mathrm{~mm}$. stage. Caudal migration then proceeds. About the fourth week, or $5 \mathrm{~mm}$. stage, the trachea is developed. At this stage the differentiation of the sixth arch into the pulmonary artery has not occurred. This takes place in the $7 \mathrm{~mm}$. embryo. It is thus unlikely that vascular patho-embryological changes are responsible for bilateral complete absence of the lungs. Few such cases have been reported (Schmit, 1893; Allen and Affelbach, 1925; Claireaux and Ferreira, 1958; Tuynman and Gardner, 1952). Unilateral agenesis is commoner and the factors producing these congenital defects exert their adverse effects at or after the $5 \mathrm{~mm}$. stage but before the $7 \mathrm{~mm}$. stage. Like bilateral complete agenesis, unilateral complete absence of the lung cannot be attributed to vascular malformations. The left lung is more frequently affected than the right. 
Most authors have described the condition as including (1) a compensatory hypertrophy of the contralateral lung, (2) accessory contralateral lobes, (3) imperceptible merging of the trachea into the bronchus, (4) an absent pleural cavity on the affected side, and (5) a rotation and mediastinal shift towards the side of the missing lungs. This case illustrates most of these points.

Further detailed reviews have been produced on the other subdivision by Jones (1955), Breton and Dubois (1957), and Ferencz (1961).

\section{DISCUSSION}

These infants are prone to respiratory infections and present most commonly with bronchopneumonia. The most frequent symptoms have been cough, wheezing, and cyanosis. Due to the absent lung, asymmetry of the chest wall is common. Tracheal and mediastinal deviation to the side of the missing lung occur frequently. Marked dullness, owing to this displacement of the mediastinum, may simulate a hydro- or pyo-thorax, especially when pyrexia exists.

Dextrocardia or situs inversus may be mistaken for a right unilateral pulmonary agenesis. The diagnosis is not easy and can only be presumptive at the end of the physical examination. It can only be clarified by means of the following ancillary investigations. The radiographs of the chest are not diagnostic and these give the appearance of a total atelectatic lung and, with a history of repeated pneumonia, may be mistaken for an empyema thoracis; this point should be stressed. Bronchoscopy is invaluable, but in critically ill children it carries a high risk. Failure to fill a bronchus at brorachography should not be assumed to be pathognomonic of an agenesis. Total obstruction of the bronchus can be produced by abnormal vascular rings or obliterated vascular structures. This should be suspected when there are other congenital cardiovascular malformations. In the newborn, angiocardiography is useful to demonstrate the vascular pattern of the lung and other cardiovascular lesions. An E.C.G. may be helpful.

A total of 120 cases of agenesis and aplasia have been collected from the literature by Minetto (1958) according to Jimenez-Martinez et al. (1965). On the other hand, Brünner and Nissen (1963), during a discussion on two cases of agenesis of the lung, noted that 150 cases were recorded at that time.

This discrepancy in the numbers quoted is probably due to a difference in classification rather than in the actual numbers found in the literature from all parts of the world. The terms agenesis, aplasia, and hypoplasia are synonymous to some authors, while to others they convey an altogether different connotation. Agenesis denotes an absent lung, without any trace of even its bronchus, as shown in our case. Aplasia represents a rudimentary bronchus with complete absence of pulmonary parenchyma. Hypoplasia is applicable to a rudimentary functionless lung with or without a normal vascular pattern. The specific use of these terms has the added advantage of indicating the precise stage in embryological development at which the teratogenic factors affect the embryo.

Field (1946) reported several cases of tem- 음 porary hypoplasia. These cases were followed by $\rightarrow$ serial radiographs, and eventually these lungs expanded fully. A case of temporary hypoplasia was discussed by Wexels (1951) in a discussion on unilateral agenesis. It is likely that cases of socalled temporary hypoplasia are really neonatal atelectases.

These congenital anomalies carry a high mortality whether or not there are multiple associated abnormalities. The mortality is $33 \%$ in the first year of life (Nicks, 1957). This figure rises to $50 \%$ before the age of 5 years (Maier and Gould, $\stackrel{\mathbb{Q}}{\unrhd}$ 1953). It is still not clear why these infants are so $\overrightarrow{\vec{B}}$ prone to a variety of respiratory illnesses. So often the repeated attacks of pneumonia culminate in the death of the neonates. Sometimes the patient appears to be progressing satisfactorily and yet dies from uncertain causes. The present case was typical; after progressing well after several ஹ্㐅 stormy periods, the baby died from causes that $\bar{\sigma}$ were not understood. Possibly the cilial and the mucous-secreting glands of the respiratory $\delta$ tract are also maldeveloped, so children with an agenetic or hypoplastic lung may have an in- o creased liability to infection as they cannot shift $\rightarrow$ their mucus. A review of the reported radiographs showed an excessive kinking of the trachea and N functioning bronchus in the majority of cases. Maldevelopment of the cartilages both structurally $N$ and numerically may be responsible for the exces- $N$ sive mediastinal shift which hinders proper drain- 음 age of the functioning lung. Most authors have 0 pointed out that children tolerate pneumonectomy $\overparen{D}$ well, and yet unilateral hypoplasia or agenesis is $\stackrel{?}{+}$ associated with a high mortality in the first years $\underline{T}$ of life.

In a comprehensive review of pulmonary $\stackrel{\varnothing}{\circledR}$ vessels and lung malformations Oyamada, Gasul, $\stackrel{\mathbb{\perp}}{\varrho}$ and Holinger (1953) studied all previously reported cases of pulmonary hypoplasia, and they 
suggested from this study that hypoplasia carried a slightly better prognosis than agenesis. They noted that of 38 hypoplastic lungs described since 1880 seven patients lived for more than 20 years and the oldest lived to 56 years. The longest survival of pulmonary developmental defect was a woman who lived to 72 years. This case was quoted by Field (1946) in her discussion on pulmonary agenesis and hypoplasia. Wexels (1951), who tabulated 87 cases of authentic agenesis of the lungs, noted that six patients lived to the ages of $37,27,52,43,41$, and 50 years.

The patient reported in this paper has certain features worth recording. This was the first case, so far as the authors are aware, to be diagnosed in life with a combined congenital tracheal stenosis, left pulmonary agenesis, and a patent ductus arteriosus.

The tracheal stenosis, only $2 \mathrm{~mm}$. in diameter compared to a normal $4 \mathrm{~mm}$., by itself carries a poor prognosis. On reviewing the literature we found only two other cases with a combined congenital tracheal stenosis and unilateral agenesis of the lung, but neither had a patent ductus arteriosus; neither survived the neonatal period. Wehr (1934) reported the necropsy on a newborn girl with stenosis of the trachea, no left bronchus, atresia of the larynx, oesophago-tracheal fistula, and a septal defect. Killingsworth and Hibbs (1939) reported the necropsy on a 10-day-old girl and described a narrowing of the trachea to $3 \mathrm{~mm}$. in diameter, a right lung agenesis, a patent ductus arteriosus, a pulmonary artery stenosis, a patent foramen ovale, an absent left kidney, and synostosis of the first, second, and third ribs.

Three other cases of unilateral lung agenesis or aplasia and a patent ductus arteriosus, but no tracheal stenosis, have been diagnosed during life. Nicks (1957) first performed a successful division of a patent ductus arteriosus on a 7-year-old girl with a left agenetic lung. Jimenez-Martinez et al. (1965) reported the second successful treatment of a case identical to that of Nicks, and Lukas, Dotter, and Steinberg (1953) reported the cardiac catheterization findings on a lifelong dyspnoeic 22 year-old man with clinical features similar to those of the above-mentioned two cases. His pulmonary hypertension $(113 / 70 \mathrm{~mm}$. $\mathrm{Hg})$ with a reversal of the shunt precluded treatment of his patent ductus arteriosus. This case stresses the need for early treatment of the patent ductus arteriosus even if it is asymptomatic. Furthermore, angiocardiography, though not performed in our case, is indicated in all cases to exclude other vascular anomalies associated with the aortic arch. During the last decade individual authors have discussed only one or other aspect of this interesting, but rare, maldevelopment. We have therefore reviewed the subject.

\section{SUMMARY}

A case of left pulmonary agenesis, congenital tracheal stenosis, and a patent ductus arteriosus and its treatment is described.

This is the fourth example of unilateral pulmonary agenesis with a patent ductus arteriosus to be diagnosed in life and the first to be treated for its additional tracheal stenosis.

It is also the first reported to undergo bilateral thoracotomy. The division of the patent ductus arteriosus and recovery from it was uncomplicated. A second thoracotomy on the right to relieve the congenital tracheal stenosis by a dermal graft was temporarily successful, and after a difficult post-operative period the patient made good progress for two months. Death occurred suddenly after two months for no obvious reason. Necropsy showed a satisfactory correction of the congenital stenosis.

The authors wish to thank Dr. D. A. J. Williamson, consultant paediatrician, for referring the case, Dr. E. Machell for the bronchogram, and Drs. D. Pearce and L. Langdon for coping with the difficult problems of anaesthesia during the two operations, the bronchoscopies, and the bronchography. They wish also to thank all the nursing staff who devoted so much time and effort to this case, which presented so many difficult nursing problems. Thanks are due to Mr. M. White for the photographs and to Miss G. Beal for secretarial assistance.

\section{REFERENCES}

Allen and Affelbach (1925). Congenital absence of both lungs. Surg. Gynec. Obstet., 41, 375.

Breton, A., and Dubois, O. (1957). Les Malformations Congénitales du Poumon. Doin, Paris.

Brünner, S., and Nissen, E. (1963). Agenesis of the lung. Amer. Rev. resp. Dis., 87, 103.

Butler, N. R., Dudgeon, J. A., Hayes, K., Peckham, C. S., and Wybar, K. (1965). Persistence of rubella antibody with and without embryopathy. Brit. med. J., 2, 1027.

Campbell, M. (1961). Place of maternal rubella in the aetiology of congenital heart disease. Ibid., 1, 691.

Claireaux, A. E., and Ferreira, H. P. (1958). Bilateral pulmonary agenesis. Arch. Dis. Childh., 33, 364.

Ferencz, C. (1961). Congenital abnormalities of pulmonary vessels and their relation to malformations of the lung. Pediatrics, 28, 993.

Field, C. E. (1946). Pulmonary agenesis and hypoplasia. Arch. Dis. Childh., 21, 61.

Finkelstein, H. (1912). Hypoplasie and Agenesie der Lunge. In Lehrbuch der Säuglings Krankheiten. Fischer's Medizin. Buchland, Berlin.

Forrester, R. M., Lees, V. T., and Watson, G. H. (1966). Rubella syndrome: escape of a twin. Brit. med. J., 1, 1403. 
Jimenez-Martinez, M., Perez-Alvarez, J. J., Perez-Trevino, C., RubioAlvarez, V., and de Rubens, J. (1965). Agenesis of the lungs with patent ductus arteriosus treated surgically: report of a case. J. thorac. cardiovasc. Surg., 50, 59.

Jones, P. (1955). Developmental defects in the lungs. Thorax, 10, 205.

Killingsworth, W. P., and Hibbs, W. G. (1939). Agenesis of the lung: review of the literature and report of a case. Amer. J. Dis. Child. 58. 571 .

Lukas, D. S., Dotter, C. T., and Steinberg, I. (1953). Agenesis of the lung and patent ductus arteriosus with reversal of flow. New Engl.J. Med., 249, 107.

Maier, H. C., and Gould, W. J. (1953). Agenesis of the lung with vascular compression of the tracheobronchial tree. J. Pediat., 43, 38.

Neill, C. A., Ferencz, C., Sabiston, D. C., and Sheldon, H. (1960), The familial occurrence of hypoplastic right lung with systemic arterial supply and venous drainage. Bull. Johns Hopk. Hosp. 107, 1 .
Nicks, R. (1957). Agenesis of the lung with persistent ductus arteriosus. Thorax, 12, 140

Oyamada, A., Gasul, B. M., and Holinger, P. H. (1953). Agenesis of the lung: report of a case, with a review of all previously reported cases. Amer. J. Dis. Child., 85, 182.

Sandoz, E. (1927). Ueber 2 Falle von 'fotaler Bronchiektasie' Berner Pathol. Inst. Fischer, Jena.

Schmit, H. (1893). Ein Fall von vollständiger Agenesie beider Lungen. Virchows Arch. path. Anat., 134, 25.

Schneider. P and Schwalbe, E (1913), Die Morphologie der Missbildungen des Menschen und der Tiere, 3, 321. Fischer, Jena.

Tuynman, P. E., and Gardner, L. W. (1952). Bilateral aplasia of the lung. Arch. Path. (Chic.), 54, 306.

Wehr, Clemens (1934), Inaugural dissertation, Gottingen. Cited by Bohnholtzer, E. (1938). Beitr. path. Anat., 101, 614.

Wexels, P. (1951). Agenesis of the lung. Thorax, 6, 171.

Wilson, J. G., and Warkany, J. (1949). Aortic-arch and cardiac anomalies in the offspring of vitamin A deficient rats. Amer. $J$. Anat., 85, 113 . 